\title{
Carnap y Husserl: a propósito de la constitución de la experiencia
}

\author{
Carlos Alberto Cardona SuÁrez \\ Escuela de Ciencias Humanas \\ Universidad del Rosario \\ carlos.cardona@urosario.edu.co
}

Resumen: En el presente artículo se desarrollan tres tareas. (i) Se presenta un mapa de posibles y antagónicas lecturas del Aufbau de Carnap y se favorece una lectura kantiana con rasgos fenomenológicos. (ii) Se identifica críticamente una dificultad central del Aufbau cuando se exige el carácter instantáneo de las experiencias elementales. (iii) Se propone una alternativa para minimizar la dificultad atendiendo algunas sugerencias propuestas por Husserl en Experiencia y juicio, aun cuando se advierte acerca de la necesidad de formular serias revisiones a la geometría conceptual del Aufbau.

Palabras clave: Aufbau, experiencia elemental, cuasianálisis, geometría conceptual

\begin{abstract}
This paper carries out three tasks. (i) It presents a map of possible and antagonistic readings of Carnap's Aufbau and suggests a Kantian reading with phenomenological features. (ii) A central difficulty of the Aufbau is identified when the instantaneous feature of the elementary experiences is demanded. (iii) A possibility of minimizing this difficulty is presented using some suggestions proposed by Husserl.
\end{abstract}

Key words: Aufbau, elementary experience, quasianalysis, conceptual geometry

\section{Introducción}

El aporte de Carnap a la filosofía puede caracterizarse como un aporte de múltiples entradas. Podemos llegar a la filosofía de Carnap preocupados ora por el problema de la inducción, ora por la sistematización de la semántica, ora por la consolidación de la sintaxis del lenguaje, ora por la naturaleza de la investigación científica, ora por la constitución de la experiencia. Por cualquiera de las vías de acceso es factible encontrarse, de primera mano y entre otros, con dos rasgos característicos fuertemente conectados. En primer lugar, la defensa de diferentes presentaciones de principios de tolerancia, todas ellas conducentes al cultivo de diferentes versiones de neutralidad en relación con las disputas clásicas entre realismos y escepticismos; y, en segundo lugar, una elocuente honestidad intelectual que condujo al autor, en varias ocasiones, a dar marcha atrás en virtud de la fuerza de lo que parecían ser poderosos argumentos en contra. 
La carta de presentación de Carnap en los debates filosóficos es, sin duda y sin que se trate de su primer escrito de importancia, su obra de 1928 Der logische Aufbau der Welt. ${ }^{1}$ La riqueza hermenéutica de dicha obra se advierte también en las múltiples posibilidades de lectura que determinan, a su vez, múltiples vías de acceso a la misma. Quiero advertir dos polaridades y señalar la posición personal que ha de determinar la aproximación del presente artículo.

Primera polarización. El Aufbau puede leerse, en primer lugar, como la culminación del proyecto empirista, proyecto orientado a desterrar cualquier posibilidad de un fundamento a priori del conocimiento; para ello, Carnap pretende usar las herramientas dispuestas por la nueva lógica, en particular la lógica de relaciones desarrollada por Russell y Frege. Ésta es la lectura recomendada por Quine, quien supone que los argumentos consignados en "Dos dogmas del empirismo" exigen una reorientación radical del proyecto; una reorientación que renuncie tanto a la clásica distinción analítico/sintético, como a la aspiración reduccionista radical. ${ }^{2}$ La lucha de Kant contra el empirismo se asentaba en la posibilidad de exhibir las proposiciones de la geometría como ejemplos de juicios sintéticos a priori. La réplica de los empiristas siempre encontró allí el principal obstáculo. El desarrollo de la lógica de relaciones hizo posible estructurar un fuerte argumento contra Kant; a saber, que es posible restituir analíticamente los juicios de la geometría, siempre que podamos disponer de relaciones como herramienta básica. Carnap, al tenor de esta recomendación de lectura, se dio a la tarea de completar el ejercicio iniciado por Bertrand Russell, ${ }^{3}$ es decir, reconstruir la totalidad de los conceptos de la ciencia fundándolos no en categorías a priori, sino en métodos constructivos apoyados en relaciones básicas. En ese orden de ideas, la epistemología ingenua del empirismo clásico debía abandonar la pretensión de ir desde los objetos teóricos a la predicción experimental, para tratar de reconstruir

${ }^{1}$ En lo sucesivo haré referencia a ella con la expresión Aufbau y la citaré por parágrafos.

${ }^{2}$ Quine exhibe el sistema del Aufbau como el intento más serio de reduccionismo radical y propone, a manera de sustituto, la defensa de una forma de holismo. Véase Quine 1951, especialmente las pp. 34-39.

${ }^{3}$ En una copia personal de Our Knowledge of the External World de Russell, al final de la tercera conferencia, donde el autor especula que su construcción "puede obtenerse a partir de los materiales más escasos por medio de los métodos lógicos de los cuales tendremos un ejemplo en las definiciones de puntos, instantes y partículas" (p. 105), Carnap escribió en el margen: "Este estrechamiento y profundización de los postulados fundamentales es mi tarea" (Friedman 1999, p. 117, n. 7).

Diánoia, vol. LV, no. 64 (mayo 2010). 
los objetos teóricos en términos de sense-data, siguiendo a Russell, o de experiencias elementales, siguiendo a Carnap.

El Aufbau también puede leerse, en segundo lugar, como un intento por culminar un proyecto de orden kantiano orientado a explicar cómo es posible restituir la objetividad del conocimiento científico si se admite como punto de partida el marco de la experiencia subjetiva individual. Esta esperanza se enuncia sin vacilación en el Aufbau de la siguiente manera:

Aun cuando el origen subjetivo de todo el conocimiento reside en los contenidos de la experiencia y sus conexiones, es posible, como el sistema constructivo mostrará, proponer un mundo objetivo intersubjetivo, el cual puede ser conceptualmente comprendido en forma idéntica por todos los observadores. (Carnap 1998, § 2)

La esperanza puede leerse así: aun cuando lo que nos es dado es enteramente subjetivo, es posible llegar a restituir la objetividad plena del conocimiento científico. Michael Friedman, uno de los defensores de tal estilo de lectura, advierte que las motivaciones epistemológicas del Aufbau se pueden entender de manera más completa si se tienen en cuenta los problemas de la tradición neokantiana, a saber, ¿cómo es posible que el proceso cognitivo transforme las sensaciones inherentemente privadas y subjetivas en una experiencia completamente objetiva susceptible de validación y verificación? ¿Y cómo hacerlo sin tener que acudir a herramientas metafísicas o psicologicistas? ${ }^{4}$

Segunda polarización. En términos técnicos, el Aufbau se propone ofrecer criterios de traducción que permitan sustituir entidades inferidas por construcciones lógicas. ${ }^{5}$ Se dice que un objeto es reducible a otros si todas las proposiciones que incluyen al primero pueden ser traducidas, sin cambiar su valor de verdad, como proposiciones que hablan únicamente acerca de los segundos (Carnap 1998, § 35). El único criterio que se exige es extensional. ${ }^{6}$ Ahora bien, con el ánimo de excluir las discusiones metafísicas que entorpecen la claridad científica,

${ }^{4}$ Friedman 1999, p. 141. Véanse también: Friedman 2000; Richardson 1992, 1998; Webb 1992; Haack 1977; Proust 1989.

${ }^{5}$ El epígrafe del Aufbau señala esta orientación. Tal epígrafe se recoge a partir de un artículo de Russell y reza así: "La máxima suprema en filosofía científica es ésta: allí donde sea posible, las construcciones lógicas deben reemplazar a las entidades inferidas" (Russell 2004, p. 121).

${ }^{6}$ Dos conceptos (funciones proposicionales) se dicen idénticos si y sólo si coinciden en los elementos que los satisfacen. 
Carnap pretende traducir todos los conceptos científicos en términos de enunciados que refieran exclusivamente a lo inmediatamente dado. En ese orden de ideas, se requiere una definición precisa de lo dado y unos criterios claros que definan las formas de ascensión que avancen a partir de lo dado hacia los conceptos construidos. Las formas de ascensión se definen a partir de los métodos de construcción lógica de Russell, ${ }^{7}$ en tanto que los elementos de la base pueden ser de naturaleza diferente: o bien objetos físicos, o bien objetos psicológicos (y entre ellos pueden encontrarse objetos psicológicos en general o autopsicológicos), o bien objetos culturales. Carnap defiende una tesis de neutralidad según la cual, en el lenguaje realista (que de hecho difiere del lenguaje lógico) podemos adoptar cualquier punto de partida inicial (objetos físicos u objetos psicológicos) y a continuación podemos construir los objetos de las otras esferas. El sistema de construcción es neutral en relación con la elección efectuada: "El lenguaje realista, que es el que las ciencias empíricas usan generalmente, y el lenguaje constructivo tienen realmente el mismo significado: ambos son neutrales en cuanto a la decisión del problema metafísico de la realidad entre realismo e idealismo" (Carnap 1998, § 52). El lenguaje lógico del sistema constructivo no requiere, pues, comprometerse con ninguna interpretación particular de lo dado (objetos físicos u objetos psicológicos). No obstante - y para efectos de la presentación y construcción del esbozo del Aufbau-, Carnap sugiere un criterio auxiliar de primacía epistémica según el cual el orden de construcción debe exhibir, también, el orden de dependencia epistémica. $^{8}$

La forma del sistema que queremos dar a nuestro esbozo del sistema constructivo - explica Carnap- está caracterizada por el hecho de que éste no sólo intenta exhibir, como cualquier forma de sistema, el orden de los objetos relativo a su reducibilidad, sino que intenta también mostrar su orden relativo a la primacía epistémica. (Carnap 1998, § 54)

${ }^{7}$ El principio básico es el principio de abstracción que reza así: "Cuando un grupo de objetos tiene aquella clase de semejanza que nosotros estamos inclinados a atribuir a la posesión de una cualidad común, el principio en cuestión muestra que el ser miembro del grupo servirá a todos los propósitos de cualidad común supuesta, y que, en consecuencia, a menos que alguna cualidad sea realmente conocida, el grupo o clase de objetos similares puede ser usado para reemplazar la cualidad común, la cual no necesita ser asumida como algo existente" (Russell 1993, p. 51).

${ }^{8}$ Un objeto es epistémicamente primario, en relación con otro, si el segundo es reconocido a través de la mediación del primero.

Diánoia, vol. LV, no. 64 (mayo 2010). 
En el trasfondo de la tesis de la neutralidad y con el ánimo de ofrecer un esbozo constructivo, Carnap aplica el criterio de primacía epistémica para adoptar como punto de partida un sistema basado en la elección de una base autopsicológica. Lo dado está constituido por mis experiencias elementales, ${ }^{9}$ siempre que tenga en cuenta las siguientes dos precauciones: (i) no exigir un sujeto que en forma paciente padece dichas experiencias; ${ }^{10}$ (ii) considerarlas al principio tal y como ellas se presentan, es decir, desprovistas de cualquier cualidad ulterior ${ }^{11} \mathrm{y}$ presentes en un flujo de experiencias.

La segunda polarización se desprende, pues, de la actitud que se adopte frente a la tesis de la neutralidad. O bien adoptamos - a pesar de la primacía epistémica - una posición austera que opta por considerar literalmente la tesis mencionada, o bien sugerimos - a pesar de la tesis de la neutralidad- que Carnap desea defender estructuralmente una forma de fenomenalismo. En el primer caso, la defensa de una lectura austera, hemos de concentrarnos en los métodos lógicos de ascensión que permiten una arquitectónica de conceptos a partir de lo dado, cualquiera que sea la interpretación adoptada. En ese orden de ideas podemos contemplar el Aufbau a la manera de una geometría (o topología) para la construcción y articulación de conceptos. Habría, entonces, dos elementos esenciales en la base de la construcción: (i) lo dado: experiencias elementales; ${ }^{12}$ y (ii) las posibles conexiones esenciales entre los elementos dados, a saber, la relación o las relaciones básicas. La propuesta de una geometría conceptual aportaría al conocimiento científico en general lo mismo que la geometría de Hilbert aportó al estudio de la geometría. Así como el modelo de Hilbert aportó un andamiaje general de posibles conexiones que después podría cobrar cuerpo en la forma de una geometría euclidiana, una geometría proyectiva, una geometría hiperbólica, etc., el modelo de Carnap habría

${ }^{9}$ En adelante, exel.

${ }^{10}$ Carnap usa el término solipsismo metodológico para referirse a esta particular contención del sistema. En ese orden de ideas, Carnap se abstiene de afirmar realidad o irrealidad en conexión con dichas experiencias y prefiere reservar para ellas la abstención del juicio a la manera de la epojé en el sentido de Husserl 1986, $\S \S 31,32$.

${ }^{11}$ En este segundo punto las experiencias elementales difieren radicalmente de los sense-data de Russell.

${ }^{12}$ En el curso de formación del sistema y tratando de copiar los métodos de construcción de la geometría al estilo de Hilbert, el único elemento extralógico lo constituye la relación fundamental, en tanto que las experiencias elementales se definen de manera implícita a partir de dicha relación (cfr. Carnap 1998, §§ 75, 109).

Diánoia, vol. LV, no. 64 (mayo 2010). 
de ofrecer un andamiaje formal de posibles conexiones que después podría cobrar cuerpo en la forma de un esbozo autopsicológico, o un esbozo físico, etc. Lo dado podría representarse por medio de puntos y las posibles parejas atadas en virtud de una (o varias) relación(es) fundamental(es) podrían darse a conocer por medio de flechas (o segmentos) que conectan los elementos relacionados. Una vez establecidas las propiedades formales de las relaciones involucradas se puede ascender a niveles superiores desplegando las estrategias que posibiliten tales propiedades. Así las cosas, dada una malla de experiencias elementales (nodos) y relaciones básicas (flechas), es posible elaborar criterios de construcción de conceptos complejos a partir de los cuales se puede concebir el lenguaje de un aparato conceptual científico, este último elaborado en forma ardua a lo largo de siglos sin contar aún con la conciencia explícita de los métodos lógicos de dicha construcción. La figura siguiente ilustra una malla, en exceso simplificada, de una posible red abstracta que bien hubiese podido modelar un flujo de experiencias elementales.

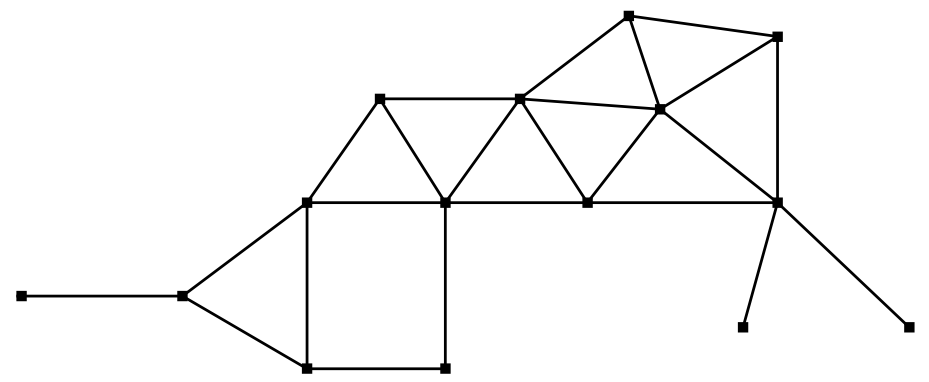

En artículos recientes Thomas Mormann (de quien hemos tomado la figura anterior) ${ }^{13}$ se ha encargado de ofrecer argumentos estimulantes para defender esta posible alternativa de interpretación del Aufbau. En palabras de Mormann:

El núcleo filosófico del Aufbau es el método lógico de constitución cuasianalítica. El cuasianálisis, sin embargo, es un método puramente estructural independiente de la naturaleza de los elementos para ser cuasianalizados. En el sistema constructivo los elementos son "átomos" o "puntos sin cualidades", como Carnap dice. (Mormann 2003, p. 62, n. 1)

${ }^{13}$ Cfr. Mormann 2003, p. 55.

Diánoia, vol. LV, no. 64 (mayo 2010). 
Esta lectura reduce - aquí reducción no es peyorativo- el Aufbau a una conceptografía formal -incluso en el sentido del Begriffsschrift de Frege- que se puede usar como una plantilla o dispositivo técnico para evaluar o posibilitar la construcción legítima de conceptos en un área especifica del conocimiento empírico. La adopción de una base autopsicológica se puede ignorar sin que el Aufbau pierda con ello su fuerza argumentativa. En ese orden de ideas, su contribución se reduce al aporte de un dispositivo técnico lógico formal comparable, quizá, al desarrollo de una teoría de la prueba o una teoría de modelos, por ejemplo.

En el segundo caso, una lectura fenomenalista, el criterio de primacía epistémica no cumple el simple papel de interfase didáctica que abre la posibilidad de ofrecer un esbozo que ejemplifica los principios de la malla geométrica, sino que introduce el problema clásico de la constitución de la experiencia y ofrece la alternativa de ocuparse de él sin verse en la obligación de aportar un fundamento trascendental (a la manera de Kant) o un fundamento psicológico (a la manera de Brentano). En ese orden de ideas, el compromiso con las bases autopsicológicas no es un compromiso secundario (prescindible), ni retórico. El lector que ingresa al Aufbau por esta puerta encuentra a su disposición nuevos caminos que se abren. Por lo pronto expongo tres posibilidades: (a) los vasos comunicantes con los teóricos de la Gestalt, ${ }^{14}$ (b) los vínculos con las preocupaciones de los círculos neokantianos, ${ }^{15}$ (c) la simbiosis con los proyectos fenomenológicos. ${ }^{16}$

La primera polarización en la interpretación del Aufbau puede, pues, sintetizarse en los siguientes términos: o (i) culminación madura del empirismo (con su consecuente marcha atrás en virtud de la crítica de Quine); o (ii) reorientación de un programa kantiano. La segunda polarización, a su vez, se sintetiza así: o (iii) construcción de una geometría conceptual abstracta en la que podemos hacer caso omiso de la interpretación de los elementos (átomos) de la base; o (iv) constitución fenomenológica de la experiencia. Si se adopta la lectura empirista (i), bien podemos optar por poner énfasis en los aspectos analíticos (lógicosintácticos) ((i) + (iii)), o en los aspectos representacionales ((i) + (iv en cualquiera de las versiones (a) o (c))). Si se adopta la lectura kantiana (ii), podemos minimizar la importancia de (iii) o reinterpretarla en un contexto subsidiario de alguna de las tres versiones de (iv). El presente artículo pretende favorecer la lectura (ii) en el ámbito de una

${ }^{14}$ Majer 2003.

${ }^{15}$ Friedman 2000; Moulines 1996.

${ }^{16}$ Sarkar 2003; Roy 2004; Beaney 2004; Ryckman 2007; Rosado 2008. 
interpretación fenomenalista (iv-c) que no le da la espalda al carácter constructivo de (iii). Se exhibirá una limitación crucial del proyecto del Aufbau que puede llegar a resolverse si se atienden algunas sugerencias de Husserl o de Peirce y se busca una forma de replantear, sin renunciar a ella, la geometría conceptual (iii). En la primera parte se presenta una síntesis del sistema constructivo del Aufbau y se advierte el aspecto crítico que se desea superar. En la segunda parte se exhiben los elementos husserlianos que podrían contribuir a la solución de la dificultad; se exhibe, también, un posible esbozo de solución y se señalan los aspectos que han de revisarse en el modelo de la geometría conceptual. Dada la diferencia radical entre las orientaciones metodológicas - $\mathrm{y}$ de hecho, filosóficas - entre Carnap y Husserl no se sugiere una propuesta de integración de los dos enfoques; se propone, más bien, conservar el espíritu y la pretensión del programa de Carnap atendiendo una sugerencia de Husserl en el ámbito de la constitución de la experiencia.

\section{El sistema constructivo y la crítica de la instantaneidad}

El propósito general del Aufbau — presentado en forma esquemáticaconsiste en ofrecer un método que permita reducir todos los conceptos útiles de la ciencia a lo inmediatamente dado, es decir, a enunciados que sólo tengan como elementos extralógicos las denominadas exel y las relaciones básicas. Una de las conjeturas centrales del tratado asegura que sólo es necesaria una relación básica, a saber, la relación recuerdo de semejanza $\left(R_{S}\right)$ (Carnap 1998, §§ 78, 82). Esta relación posee las siguientes propiedades formales: es antirreflexiva, antisimétrica, y sólo en ciertos casos es transitiva. Esto la hace una excelente candidata para constituir en forma provisional el orden temporal del flujo de nuestras experiencias. Se dice que el orden es provisional porque la falta de transitividad en todos los casos - y, a partir de ello, la posibilidad de que se presenten hiatos en el flujo de nuestras vivencias- no permite imponer un orden definitivo a no ser que se haya completado la construcción del sistema, en particular sólo en el momento en que se haya garantizado el espacio para la introducción de leyes físicas. En el lenguaje realista de la psicología, la relación $R_{S}$ se puede definir así: $x R_{S} y$ si y sólo $\operatorname{si}^{P} x, y$ son experiencias elementales tales que un recuerdo de $x$ es parcialmente semejante a la experiencia actual $y^{P} \cdot{ }^{17} R_{S}$ cumple tres papeles funda-

\footnotetext{
${ }^{17}$ Usamos el recurso tipográfico ideado por Carnap de encerrar en superíndices $P$ aquellos enunciados que se formulan en el lenguaje realista (en este caso psicológico) y en superíndices $C$ aquellos enunciados que se formulan en el lenguaje lógico (el lenguaje preferido del sistema).
}

Diánoia, vol. LV, no. 64 (mayo 2010). 
mentales: (i) a partir de ella se definen las exel como todos aquellos elementos que caen bajo la relación $R_{S}$, así $R_{S}$ es el único elemento extralógico del sistema (Carnap 1998, §§ 75, 108, 109); (ii) $R_{S}$ introduce provisionalmente un orden temporal primitivo (Carnap 1998, §§ 87, 120 ); (iii) $R_{S}$ introduce un primer concepto ordenador a partir del cual se puede iniciar el ejercicio de síntesis de la multiplicidad diversa presente en el flujo de nuestras exel (Carnap 1998, §§ 75, 83). El ascenso a niveles superiores se puede adelantar si contamos con relaciones de equivalencia (en ese caso, las clases de equivalencia constituyen los conceptos del tipo siguiente) o, en su defecto, con relaciones reflexivas y simétricas (en este caso, el ascenso se produce gracias a un complejo algoritmo denominado cuasianálisis). Dado que $R_{S}$ no reúne ninguna de las dos condiciones anteriores, Carnap procede a forzar una nueva relación $P_{S}$ (semejanza parcial) que posibilita el cuasianálisis. Esta relación se define en los siguientes términos: $x P_{S} y$ si y sólo si $x R_{S} y$ o $y R_{S} x$ o $x=y$ (Carnap 1998, $\S \S 77,110$ ).

$P_{S}$ es reflexiva, simétrica y, en principio, no tiene que ser transitiva. El cuasianálisis permite aislar clases de exel a las que podemos atribuir un mismo cuasiconstituyente común. Las exel han de ser, por principio, elementos inanalizables (Carnap 1998, § 68), de suerte que no podemos hablar de componentes o constituyentes de ellas. No obstante, el ejercicio del cuasianálisis permite agrupar exel en una misma clase a través de un procedimiento complejo de síntesis. ${ }^{18}$ Primero se construyen los círculos de semejanza como las clases que satisfacen dos criterios esenciales, a saber: (i) cada uno de los elementos de uno de dichos círculos se relaciona con todos los miembros de él por medio de la relación $P_{S}$; (ii) si un elemento se relaciona por medio de $P_{S}$ con todos los miembros del círculo, este elemento debe pertenecer a él (Carnap 1998, $\S \S 80,111)$. Dado que $P_{S}$ no es transitiva, son de esperar múltiples y complejos traslapes entre los círculos de semejanza. A continuación se construyen las clases de cualidad como las subclases de círculos de semejanza más extensas que ya no pueden ser traslapadas esencialmente por otro círculo de semejanza (Carnap 1998, § 81, 112). ${ }^{19}$

Entre las clases de cualidad se define la relación de semejanza Sim de la siguiente manera: $\alpha \operatorname{Sim} \beta$, si y sólo si, $\alpha$ y $\beta$ son clases de cualidad tales que cada elemento $x$, que pertenece a $\alpha$, mantiene la relación $x P_{S} y$

${ }^{18}$ Carnap insiste en que el cuasianálisis es un método de síntesis presentado en la jerga del análisis (Carnap 1998, § 74).

${ }^{19}$ Dos círculos de semejanza se traslapan esencialmente si la intersección contiene más de la mitad de elementos del círculo más numeroso; en caso contrario, el traslape se considera accidental.

Diánoia, vol. LV, no. 64 (mayo 2010). 
con cada elemento y que pertenece a $\beta$. Ahora bien, $\alpha$ y $\beta$ pertenecen a la misma modalidad sensorial si y sólo si existe una cadena de clases tales que $\alpha \operatorname{Sim} . . . \operatorname{Sim} \beta$ (Carnap 1998, §§ 85, 114, 115). Las modalidades sensoriales difieren en el número de dimensión asignado. Así, la modalidad visual es 5-dimensional (dos dimensiones asignadas a la constitución del espacio del campo visual y tres dimensiones asignadas a la constitución del campo cromático (matiz, brillo y saturación)) (Carnap 1998, §§ 86, 115, 117, 118, 119).

El sistema constructivo de Carnap introduce un giro copernicano en la definición del concepto sensación. Una sensación es un par ordenado constituido por una exel y una clase de cualidad a la cual pertenece la exel en mención: $S={ }_{\text {Def }}\left(\right.$ exel $\left._{n}, \alpha\right)$, donde exel $_{n}$ pertenece a $\alpha$. La sensación no es un rasgo psicológico atribuible como estado a un sujeto empírico, sino que es un concepto construido a partir de la multiplicidad de nuestro flujo de experiencias una vez sometido al criterio ordenador y de síntesis que provee $R_{S}$. Así las cosas, ${ }^{\mathrm{P}}$ la cualidad es un constituyente de la experiencia ${ }^{\mathrm{P}}$, mientras que ${ }^{\mathrm{C}}$ la experiencia es un elemento de la cualidad $^{\mathrm{C}}$ (Carnap 1998, §§ 93, 116). En el sistema no se construyen las cualidades a partir de las sensaciones, sino éstas a partir de las primeras. En palabras de Carnap: "Una experiencia individual, tomada por sí misma, es inanalizable. Las experiencias, tomadas como una multiplicidad, pueden ser comparadas y ordenadas, y únicamente a través de este orden resultan los (cuasi) constituyentes de las experiencias individuales" (Carnap 1998, § 93). Así las cosas, dos sensaciones se dicen simultáneas si las experiencias elementales que conforman dichos pares son idénticas. Supongamos que una exel $e_{n}$ de mi flujo pertenece a la clase $\alpha_{i}\left({ }^{\mathrm{P}}\right.$ mancha rojo carmesí en el vértice superior derecho de mi campo visual ${ }^{\mathrm{P}}$ ) y a la clase $\beta_{k}$ ( ${ }^{\mathrm{P}}$ nota la ajustada al timbre de una guitarra $\mathrm{P}^{\mathrm{P}}$ ), en ese caso puedo construir las dos sensaciones simultáneas $S_{1}=\left(e_{n}, \alpha_{i}\right), \mathrm{y} S_{2}=\left(e_{n}, \beta_{k}\right)$.

Las experiencias elementales reúnen dos rasgos lógicos que, aunque indispensables, no están hermanados en el sistema constructivo. Las exel son, en primer lugar, inanalizables. Se definen de manera implícita a partir de $R_{S}$ y, por esa misma razón, no pueden definirse en términos de elementos constituyentes (las cualidades, como hemos advertido, no son rasgos descriptivos de las exel). Sólo así pueden concebirse a la manera de puntos en una geometría conceptual. Las exel son, en segundo lugar, instantáneas. Dos exel no pueden darse simultáneamente, pues ello supondría la posibilidad de establecer constituyentes en una experiencia elemental contradiciendo el hecho de haberlas asumido inanalizables. En ese orden de ideas, es posible establecer una corres-

Diánoia, vol. LV, no. 64 (mayo 2010). 
pondencia uno a uno entre los puntos de una recta - ésta simula la marcha del tiempo-y las experiencias elementales. En la construcción del sentido visual, este argumento es de vital importancia para distinguir los aspectos relacionados con la ubicación espacial de una mancha coloreada en nuestro campo visual (dos dimensiones) de los aspectos relacionados con el matiz, la saturación y el brillo de la misma (tres dimensiones). También es vital para la coordinación de experiencias elementales distribuidas en un arreglo unidimensional con las coordenadas temporales de una estructura 4-dimensional de eventos (espacio de Minkowski), coordinación indispensable para la construcción de los objetos físicos (Carnap 1998, §§ 125-128).

El carácter instantáneo de las exel se impone sin justificación alguna; es algo que admitimos sin detenernos a pensar en sus implicaciones. Aun así, la relación $R_{S}$, en su formulación realista, introduce la comparación entre una exel (que podemos concebir a la manera de un fogonazo) y un recuerdo de un fogonazo anterior. El sistema constructivo de Carnap exige que podamos comparar cualquier exel con el recuerdo de otra exel de manera instantánea y sin que podamos reconocer constituyentes en la primera. Si este reconocimiento no es instantáneo, la primera exel ya habría dado paso a una representación debilitada de ella misma. ¿Por qué hemos de aceptar que el fogonazo y el recuerdo de otro fogonazo sean instantáneos y simultáneos cuando al mismo tiempo se nos niega la posibilidad de ver el recuerdo como un ${ }^{\mathrm{P}}$ constituyente psicológico del fogonazo actual ${ }^{\mathrm{P}}$ ? (recuérdese que la simultaneidad está definida entre sensaciones y no entre exel). No veo razones epistemológicas para atarnos a esa exigencia. En otras palabras, debemos encontrar métodos constructivos que nos permitan hallar en una exel un ${ }^{\mathrm{P}}$ componente psicológico ${ }^{\mathrm{P}}$ que se pueda interpretar como el recuerdo de otra exel anterior. Constato la presencia inmediata de una ${ }^{\mathrm{P}}$ mancha verde en cierto sector de mi campo visual ${ }^{\mathrm{P}}$, evoco el recuerdo de ${ }^{\mathrm{P}}$ una mancha verde en mi campo visual en un momento anterior que no logro precisar ${ }^{\mathrm{P}}$, constato las ${ }^{\mathrm{P}}$ vecindades cromáticas entre las manchas ${ }^{\mathrm{P}}$ y después de hacer intervenir niveles muy elevados del sistema constructivo admito que estoy frente a un ${ }^{\mathrm{P}}$ reconocimiento de la presencia cercana a mi cuerpo del mismo árbol que abandoné ayer en mi jardín ${ }^{\mathrm{P}}{ }^{20}$

El problema se puede plantear en los siguientes términos. Por un lado, la constitución de la experiencia al estilo de Carnap (en particular, la construcción de cualidades) exige tener a la mano la relación $R_{S}$

${ }^{20}$ Cfr. Cardona 2006.

Diánoia, vol. LV, no. 64 (mayo 2010). 
(recuerdo de semejanza). Por otra parte, el carácter inanalizable de las exel exige que ellas se den a la manera de un fogonazo - es decir, no transcurren en el tiempo-. Si hemos de poder comparar una vivencia actual con una copia degradada de una vivencia anterior - pues en ello se fundamenta $R_{S}$-, hemos de matizar la exigencia del carácter inanalizable de las exel. Si insistimos en el carácter instantáneo de las exel, hemos de adelantar la síntesis que permite la construcción carnapiana a partir de una relación diferente de $R_{S}$.

En lo que sigue aceptaré la recomendación heurística del solipsismo metodológico y el espíritu del método constructivo de Carnap, pero presentaré argumentos que sugieren una revisión de dichos métodos de tal manera que se pueda sugerir que lo que nos es dado inicialmente son vivencias que no restringiremos a instantes de tiempo y que pueden darse en tiempos traslapados. ${ }^{\mathrm{P}}$ Ante mi campo visual aparece una danza de configuraciones cambiantes en un trasfondo de configuraciones moderadamente estables; en forma paralela, atiendo también al variado espectro de ruidos que compiten en mi campo auditivo por ganar mi atención; dejo pasar las fragancias; esporádicamente advierto ciertas cinestesias; a veces atiendo ciertas punzadas en mi estómago; de repente evoco acontecimientos que se resisten a abandonar algún lugar de mi conciencia, puede ocurrir que algunos de estos acontecimientos incorporen sonidos fantasmales; es factible que me ocupe también de alguna proyección hacia el futuro de suerte que me vea haciendo premoniciones o adivinanzas ${ }^{\mathrm{P}}$. Todas estas vivencias se traslapan en una secuencia aleatoria; no se suceden una detrás de la otra. Ninguna de ellas es un fogonazo matemático, cada una de ellas transcurre. Algunas son tan breves que no nos incomoda para ellas la noción de fogonazo. ${ }^{21}$

La sugerencia de incorporar vivencias temporalmente traslapadas, que aquí presento, tiene dos fuentes. De un lado la obra de Peirce y de otro lado la constitución de la experiencia en Husserl. Comentaré ligeramente la idea seminal en Peirce. El filósofo estadounidense tenía buenas razones para sentirse incómodo con el carácter granulado de los acontecimientos mentales:

Dos ideas no están presentes en el mismo estado de conciencia, y en consecuencia es posible que no puedan ser comparadas. Decir en consecuencia que son semejantes tan sólo sugiere que un poder oculto en las profundidades del alma nos fuerza a conectarlas en nuestros pensamientos después de que ellas ya no están presentes. (Peirce 1992, p. 313)

${ }^{21}$ Cfr. ibid.

Diánoia, vol. LV, no. 64 (mayo 2010). 
Para apoyar la idea de estados de conciencia que se extienden en el tiempo, Peirce sugiere comparar el caso con una superficie que es roja en una parte y azul en la otra. Cuando preguntamos por el color de la frontera que separa las dos partes, no debemos sentir más inclinación a responder "roja" que a responder "azul". El argumento se desarrolla de la siguiente manera: asignamos colores a superficies, no a puntos individuales; en ese sentido, en el caso límite, el color de un punto es el color de las vecindades que lo contienen; ahora bien, como cualquier vecindad que contiene algún punto de la frontera ha de estar en parte en la zona roja y en parte en la zona azul, hemos de concluir que a los puntos de la frontera les viene bien una dualidad de colores. Los colores rojo y azul conviven simultáneamente en la frontera. He aquí la conclusión de Peirce:

De igual manera [refiriéndose al ejemplo de los colores] encontramos necesario sostener que la conciencia ocupa esencialmente un tiempo; y lo que es presente a la mente en cualquier instante ordinario es lo que es presente durante un lapso en el cual este instante ocurre. Entonces, el presente es mitad pasado y mitad porvenir. (Peirce 1992, p. 322)

En ese orden de ideas, si no queremos que el recuerdo de semejanza esté anclado en un poder oculto en las profundidades del alma hemos de abrir espacio a la posibilidad de vivencias traslapadas. Husserl también considera esa posibilidad en su peculiar reflexión en torno a la constitución de la experiencia, y de ello me ocuparé en la siguiente sección.

\section{A propósito de la explicación en Husserl y las modificaciones al programa de Carnap}

Pocos meses después de la muerte de Husserl en 1938, la editorial Academia de Praga publicó algunos escritos del filósofo bajo el título Experiencia y juicio. ${ }^{22}$ El propósito de Husserl era esclarecer la esencia del juicio predicativo atendiendo su pretendido origen antepredicativo. Husserl quería poner en evidencia que ya hay un aporte lógico en niveles o estratos que la tradición ha desconocido. Estos aportes están ya presentes en el ámbito de la mera receptividad (pasividad originaria); es decir, en el ámbito que alude a la conciencia de la presencia corpórea de objetos físicos espaciales e individuales. El concepto de objeto desempeña papeles muy diferentes en los programas de Carnap y de Husserl. En el caso de Husserl, la experiencia se define como referencia directa a

${ }^{22}$ En adelante lo citaré por parágrafos. 
lo individual que, no obstante, exige una operación objetivadora del yo. Ello no supone actuar en un mero caos de datos, sino dirigir la atención hacia objetos que se destacan en tanto residen allí en un horizonte diluido de objetos. Este poder seleccionar el objeto en un horizonte vago es una precondición para reconocer después las operaciones lógicas básicas en el ámbito antepredicativo. En el caso de Carnap, al contrario, el objeto, en cuanto objeto físico individualizado, se reconoce como tal en niveles muy avanzados del proceso constructivo. ${ }^{23}$ Lo dado en la propuesta de Carnap es una suerte de información absolutamente confusa en la que sólo se puede llegar a hablar de individualidades físicas una vez se han desplegado los métodos de construcción lógica hasta niveles muy elevados. De hecho, ello es posible gracias a la aplicación de estrategias de agrupación previamente concebidas. La lógica de relaciones, explícitamente reconocida, es el instrumento que hace posible la construcción final del objeto individual. En el caso de la constitución de la experiencia a la manera de Husserl, el hecho de destacar el objeto en el fondo de un horizonte vago es una precondición para la consolidación de una lógica de relaciones.

Todo objeto, en el programa de Husserl, se da en un horizonte desde el cual reclama la atención del sujeto que se dirige a él. El ejercicio de poner en primer plano el destacar un objeto en el horizonte está siempre acompañado de una intención de expectativa (Husserl 1980, § 21), la cual puede verse frustrada de golpe cuando se produce un acercamiento intencional -es éste, precisamente, el origen antepredicativo de la negación-; o puede ocurrir también que antes de la negación se presente de golpe un modo transitorio caracterizado por la vacilación. Allí donde no se haya impuesto la negación o no haya aún lugar para la vacilación, puede surgir el interés por la contemplación explicativa del objeto.

La explicación —aclara Husserl— es un penetrar de la tendencia del interés perceptivo en el horizonte interno del objeto. En el caso de una efectuación no obstruida del interés, se cumplen las expectativas protencionales y el objeto resulta ser en sus propiedades aquello que estaba anticipado, sólo que se convierte ahora en un estar dado original. (Husserl $1980, \S 22)$

${ }^{23}$ No obstante, conviene diferenciar el objeto físico individual, que exige una construcción en niveles avanzados, de la noción de objeto en cuanto categoría lógica. La palabra objeto alude a aquello acerca de lo cual se puede formular una aseveración. Cfr. Carnap 1998, § 1.

Diánoia, vol. LV, no. 64 (mayo 2010). 
El ejercicio de la explicación, que consiste en una contemplación detenida y minuciosa del objeto en sus horizontes, exige que él pueda permanecer de alguna manera aprehendido mientras se adelantan las pesquisas de comparación en las que la conciencia se dirige a otros objetos sin perder de vista el original.

Cuando el yo se vuelve hacia un nuevo objeto, mientras el primero permanece aún asido, ello puede ocurrir o de modo que el primero todavía perdure, esté dado todavía en su duración, o de modo que él mismo ya no esté dado originaliter [...], pero que aún se mantenga asido en su desaparición retencional durante la vuelta hacia el nuevo objeto. (Husserl 1980, $\S 23 b)$

Y más adelante:

Resulta entonces que la conciencia de un presente concreto encierra en sí la conciencia de un lapso retencional del pasado y que, al llegar a su término el presente concreto, le tiene que seguir un pasado retencional, concreto y fluyente. [...] O sea, que a la conciencia de un presente concreto le corresponde no sólo el lapso retencional del pasado, sino de igual modo el futuro protensional, si bien totalmente vacío. (Husserl 1980, § 23b)

La conciencia que se dirige a un objeto con intención explicativa debe sumergirse en un flujo de vivencias que implica el mantener asido, el mirar retrospectivo y el divisar protensional. Hay, pues, una riqueza de traslapes temporales inevitables e imprescindibles. A diferencia de Husserl, Carnap concibe la experiencia elemental como un átomo muerto, un elemento a la espera de ser enriquecido con las síntesis que posibilitan los diferentes niveles de cuasianálisis. Pero el cuasianálisis requiere el recuerdo de semejanza que, a su vez, sólo podría adelantarse si se asegura, de antemano, cierta facultad retencional. En otras palabras, el ejercicio de síntesis predicativa que minuciosamente adelanta el sujeto carnapiano debe fundar sus posibilidades de éxito en una práctica prepredicativa enriquecida con la ineludible presencia de traslape de vivencias elementales, siempre que se renuncie a la exigencia de instantaneidad de las exel. Todo parece indicar que Carnap no ha logrado llegar al origen primitivo de la constitución de la experiencia, pues la efectividad del recuerdo de semejanza exige el traslape temporal de exel. En el otro polo de la tensión, Husserl parece dirigirse a objetos en algún sentido ya reconocidos como tales, ha de poder advertir en sus horizontes el reconocimiento delimitado de objetos. Carnap, por su parte, constituye el concepto de objeto. Su marco de experiencia 
fenomenológica original no contempla categorías cercanas a la noción de objeto. Así las cosas, todo parece indicar que Husserl no ha logrado llegar al origen primitivo de la experiencia, pues en primer plano ya hay que suponer objetos destacables en el horizonte.

El punto de partida de Carnap, la relación recuerdo de semejanza, parece exigir, a mi juicio, que se le complemente con algo parecido al origen antepredicativo asociado con las fuentes de las asociaciones. Citemos in extenso a Husserl para divisar el enriquecimiento potencial que podría recibir el programa de Carnap si se logran incorporar estos sugestivos elementos del programa de Husserl:

Sólo mediante el despertar asociativo pueden relacionarse unos con otros los recuerdos separados e insertarse, durante el retroceso, miembro por miembro, en una conexión intuitiva del recuerdo. Es decir, una vez despertados los recuerdos mediante la asociación, pueden luego ser ordenados dentro de un nexo temporal y determinarse el antes y el después [...]. De lo mismo o de lo semejante, la tendencia va hacia un pleno recuerdo nuevo, y ya desde antes de que algo aparezca realmente en el recuerdo, "lo que se recuerda" tiene una peculiar "intención hacia algo igual o semejante que retrocede hacia el pasado"; recuerda a algo semejante que, sin embargo, no es una nada vacía, sino que está en la conciencia de manera análoga a como lo está el horizonte, sumergido hacia atrás, de lo que acaba de ser intuitivo o como permanece lo que recién ha sido intuitivo de alguna manera oscura en el horizonte de lo que es todavía realmente intuitivo. (Husserl 1980, § 42b)

En síntesis, el proyecto de Carnap orientado a constituir la experiencia con base en la relación recuerdo de semejanza se avizora como un fracaso en la medida en que mantenga la exigencia del carácter instantáneo de las exel. En forma natural parece más prometedor adelantar el programa admitiendo experiencias elementales traslapadas en el flujo temporal tal y como se insinúa en orientaciones tan disímiles como las de Peirce y de Husserl. Por otra parte, el programa de Carnap es más parco en sus compromisos que los programas de Peirce o de Husserl. No hay en dicho programa, gracias al solipsismo metodológico que se practica, la exigencia de una conciencia que elabore la síntesis. Tampoco es necesario suponer previamente una noción debilitada de objeto individual y tampoco se exige un compromiso ontológico con alguna forma de realismo, como de hecho ocurre en el programa de Peirce. En ese orden de ideas conviene adelantar un programa de constitución de la experiencia —orientado por los criterios metodológicos de Carnap- que logre incorporar experiencias elementales traslapadas (a

Diánoia, vol. LV, no. 64 (mayo 2010). 


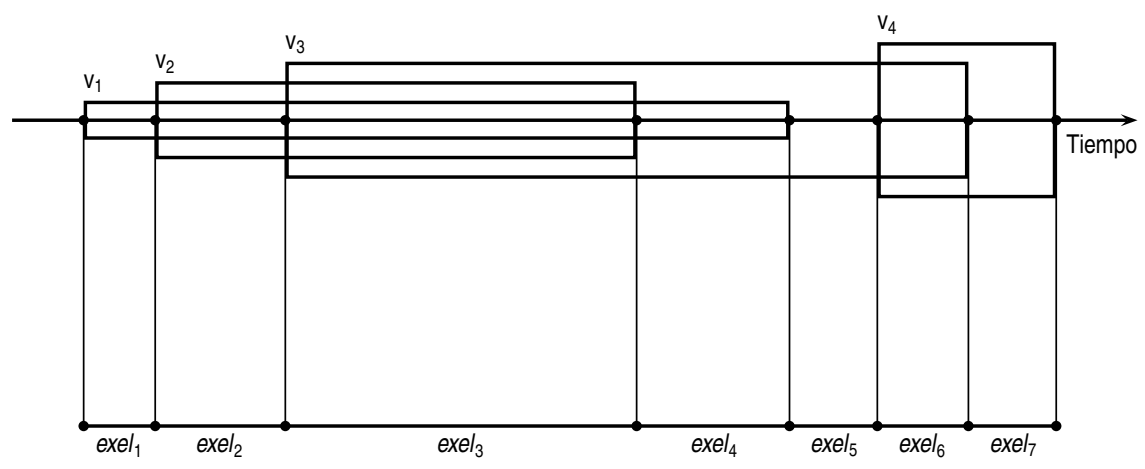

la manera de Husserl o de Peirce) para hacer posible la consolidación de la relación recuerdo de semejanza. No obstante, dicha empresa impone reformular técnicamente la base de la geometría conceptual que exige concebir las exel a la manera de puntos.

En un artículo previo he presentado los esbozos de lo que podría llegar a constituir la base inicial para un proyecto de investigación en esa dirección. ${ }^{24}$ A continuación sintetizaré de manera breve algunos de los elementos centrales. Postularé que una exel, que ha de reunir lo más cercano a un acontecimiento mental fulminante, puede concebirse a partir de un traslape enmarañado de vivencias transcurridas en segmentos de tiempo que contienen en sí el breve lapso en el que transcurre la experiencia elemental. Con el gráfico de esta página ilustro la idea que orienta la reflexión. Las vivencias identifican lo que nos es dado; cada vivencia transcurre en un periodo para el cual, en principio, se puede fijar una fecha de inicio y otra de finalización; las vivencias pueden traslaparse o no en las fechas que hemos mencionado. La gráfica ilustra las fechas asignadas a cuatro vivencias que se traslapan temporalmente de diversas maneras. Una exel se puede concebir concentrada en un segmento de tiempo que está determinado por las fechas asignadas a las vivencias. Una exel implica el traslape de varias vivencias -éstas se pueden identificar como sus componentes primarias-, de tal manera que no haya otra vivencia que se traslape a cada uno de sus componentes. ${ }^{25}$

${ }^{24}$ Cardona 2006, pp. 32-35.

${ }^{25}$ Aquí estoy parafraseando el intento de Russell orientado a definir un instante de tiempo. "Un 'instante', tal como pretendo definir el término, es una clase de sucesos que tiene las dos propiedades siguientes: 1 ) todos los sucesos de la clase se traslapan; 2) ningún suceso externo a la clase se traslapa con todos los miembros de ésta" (Russell 1983, p. 280). 
El segmento de tiempo en el que transcurre una exel es aquel durante el cual a las vivencias que constituyen la exel no se traslapa una nueva vivencia. Así las cosas, para el caso anterior se pueden identificar siete segmentos en los cuales es factible concebir igual número de exel. Usaremos paréntesis cuadrados para reconocer las vivencias que componen una exel. Conviene advertir que la relación entre vivencias y exel es una relación del todo a las partes y no se puede concebir una exel como una clase cuyos elementos son las vivencias. ${ }^{26}$

$$
\begin{array}{ll}
\text { exel }_{1}: & {\left[v_{1}\right]} \\
\text { exel }_{2}: & {\left[v_{1}, v_{2}\right]} \\
\text { exel }_{3}: & {\left[v_{1}, v_{2}, v_{3}\right]} \\
\text { exel }_{4}: & {\left[v_{1}, v_{3}\right]} \\
\text { exel }_{5}: & {\left[v_{3}\right]} \\
\text { exel }_{6}: & {\left[v_{3}, v_{4}\right]} \\
\text { exel }_{7}: & {\left[v_{4}\right]}
\end{array}
$$

Ahora imaginemos un flujo particular que nos gustaría reconocer como una cadena de recuerdos. Una cadena de vivencias $v_{1} \ldots v_{n}$ se denomina una cadena de recuerdos si se reúnen dos condiciones, una formal y otra psicológica; en primer lugar, si cada $v_{i}$ se traslapa en el tiempo con $v_{i+1}$ y cada $v_{i}$ tiene la relación de semejanza parcial con $v_{i+1}$; y, en segundo lugar, si una tensión psicológica nos conduce a postular que $v_{1}$ ha de ser parcialmente semejante con $v_{n}$. En el primer caso exigimos que las parejas de vivencias que se comparan sean simultáneas en el sentido de Husserl; y en el segundo caso imponemos, a manera de conjetura, la transitividad de la semejanza parcial entre vivencias que, aunque distantes en el tiempo, se pueden encadenar en un único flujo de vivencias. Imaginemos tres vivencias $v_{1}, v_{2}$ y $v_{3}$ encadenadas como exige la condición formal. Imaginemos que la primera es parcialmente semejante y se traslapa temporalmente con la segunda; y entre tanto, la segunda es parcialmente semejante y se traslapa con la tercera. Dado que no sostenemos identidad alguna entre ellas, bien podría ocurrir que la primera y la tercera no tuviesen ningún parecido de familia. Sin embargo, cuando una tensión psicológica nos induce a reconocer algún parecido de familia entre la primera y la tercera, podemos imaginar que ellas forman parte de los extremos de una cadena de recuerdos.

${ }^{26}$ A propósito de la diferencia entre una clase y un todo, el lector puede seguir la juiciosa distinción que sugiere Carnap en los parágrafos 36-37 del Aufbau. Una clase no está compuesta por sus elementos, mientras que un todo estructurado no puede concebirse como una clase a partir de sus constituyentes.

Diánoia, vol. LV, no. 64 (mayo 2010). 
Ahora ofreceremos una definición de semejanza parcial entre dos experiencias elementales. Sean $x, y$ dos exel, diremos que $x P_{S} y$ si existe una cadena de recuerdos $v_{1} \ldots v_{n}$ tal que para alguna vivencia $v_{\alpha}$ que forma parte de $x$ y alguna vivencia $v_{\beta}$ que forma parte de $y$, ocurre que $v_{1} P_{S} v_{\alpha}$ y $v_{n} P_{S} v_{\beta}$. De la definición se infiere que si $v_{1} \ldots v_{n}$ es una cadena de recuerdos y $v_{1}$ forma parte de la experiencia elemental $x$, en tanto que $v_{n}$ forma parte de la experiencia elemental $y$, debe ocurrir, entonces, que $x P_{S} y$. La relación $P_{S}$ entre exel es reflexiva y simétrica. No obstante lo anterior, $P_{S}$ no es necesariamente una relación transitiva. A partir de este punto es posible iniciar el cuasianálisis a la manera de Carnap.

Para finalizar conviene advertir de antemano una de las dificultades que se avizora hacia el futuro. La constitución del espacio bidimensional del campo visual exige que no haya experiencias elementales simultáneas, toda vez que tal construcción se apoya en el reconocimiento de que no puede haber simultáneamente dos colores diferentes en la misma región del espacio. ${ }^{27} \mathrm{Si}$ admitimos la posible simultaneidad de experiencias elementales se hará necesario modificar o adecuar la construcción de lugares en el campo visual. Ésta, sin embargo, es una dificultad que exige un tratamiento más complejo y extenso.

\section{BIBLIOGRAFÍA}

Awodey, S. y C. Klein (comps.), 2004, Carnap Brought Home. The View from Jena, Open Court, Chicago.

Beaney, M., 2004, "Carnap's Conception of Explication: from Frege to Husserl", en Awodey y Klein (comps.), Carnap Brought Home. The View from Jena, pp. 117-150.

Bonk, T. (ed.), 2003, Language, Truth and Knowledge. Contributions to the Philosophy of Rudolf Carnap, Kluwer Academic Publishers, Dordrecht.

Cardona, C., 2006, "A propósito de la falta de continuidad de las experiencias elementales en el Aufbau de Carnap", Praxis Filosófica, no. 23, pp. 21-38.

Carnap, R., 1998, Der logische Aufbau der Welt, Felix Meiner Verlag, Hamburgo. [Versión en castellano: La construccion logica del mundo, trad. Laura Mues de Schrenk, Instituto de Investigaciones Filosóficas-UnAM, México, 1988.]

Cirera, R., A. Ibarra, y T. Mormann (comps.), 1996, El programa de Carnap, Ediciones del Bronce, Barcelona.

Friedman, M., 2000, A Parting of the Ways. Carnap, Cassirer and Heidegger, Open Court, Chicago.

_ 1999 , Reconsidering Logical Positivism, Cambridge University Press, Cambridge.

${ }^{27}$ Cfr. Carnap 1998, §§ 88, 117.

Diánoia, vol. LV, no. 64 (mayo 2010). 
Friedman, M. y R. Creath (comps.), 2007, The Cambridge Companion to Carnap, Cambridge University Press, Cambridge.

Haack, S., 1977, "Carnap's Aufbau: Some Kantian Reflections", Ratio, vol. 19, pp. 170-176.

Husserl, E., 1986, Ideas relativas a una fenomenología pura y una filosofía fenomenológica, trad. José Gaos, Fondo de Cultura Económica, México.

— 1980, Experiencia y juicio, trad. Jas Reuter, Universidad Nacional Autónoma de México, México.

Majer, U., 2003, “Carnap's Übernahme der Gestalttheorie in den Aufbau im Lichte heutiger, vor allem computionaler Theorien des Sehen", en Bonk (comp.), Language, Truth and Knowledge. Contributions to the Philosophy of Rudolf Carnap, pp. 65-78.

Morman, T., 2007, "Geometrical Leitmotifs in Carnap's Early Philosophy", en Friedman y Creath (comps.), The Cambridge Companion to Carnap, pp. 4364.

— 2004, "A Quasi-analytical Constitution of Physical Space", en Awodey y Klein (comps.), Carnap Brought Home. The View from Jena, pp. 79-100.

— 2003, "Synthetic Geometry and Aufbau", en Bonk (comp.), Language, Truth and Knowledge. Contributions to the Philosophy of Rudolf Carnap, pp. 45-64.

Moulines, U., 1996, "Las raíces epistemológicas del Aufbau de Carnap", en Cirera, Ibarra y Mormann (comps.), El programa de Carnap. Ciencia, lenguaje, filosofía, pp. 45-74.

Peirce, Ch.S., 1992, "The Law of Mind", en Nathan Houser y Christian Kloesel (comps.), The Essential Peirce. Selected Philosophical Writings, vol. 1, Indiana University Press, Bloomington/Indianapolis, pp. 312-333.

Proust, J., 1989, Questions of Form. Logic and the Analytic Proposition from Kant to Carnap, trad. Anastasios Albert Brenner, University of Minnesota Press, Minneapolis.

Quine, W.V.O., 1951, "Two Dogmas of Empiricism", Philosophical Review, vol. 60, pp. 20-43. [Versión en castellano: "Dos dogmas del empirismo", en Luis Valdés (comp.), La búsqueda del significado, trad. Manuel Sacristán, Madrid, Tecnos/Universidad de Murcia, 1991, pp. 220-243.]

Richardson, A., 1998, Carnap's Construction of the World, Cambridge University Press, Cambridge.

_ 1992 , "Logical Idealism and Carnap's Construction of the World", Synthese, vol. 93, pp. 59-92.

Rosado, G., 2008, The Young Carnap's Unknown Master. Husserl's Influence on Der Raum and der logische Aufbau der Welt, Ashgate, Hampshire.

Roy, J.M., 2004, "Carnap's Husserlian Reading of the Aufbau", en Awodey y Klein (comps.), Carnap Brought Home. The View from Jena, pp. 41-62.

Russell, B., 2004, "The Relation of Sense-Data to Physics", en Mysticism and Logic, Dover, Nueva York, pp. 113-141.

_ 1993 , Our Knowledge of the External World, Routledge, Londres.

—_, 1983, El conocimiento humano, trad. Néstor Míguez, Orbis, Barcelona.

Diánoia, vol. LV, no. 64 (mayo 2010). 
Ryckman, T., 2007, "Carnap and Husserl”, en Friedman y Creath (comps.), The Cambridge Companion to Carnap, pp. 81-105.

Sarkar, S., 2003, "Husserl's Role in Carnap's Der Raum", en Bonk (comp.), Language, Truth and Knowledge. Contributions to the Philosophy of Rudolf Carnap, pp. 179-190.

Webb, J., 1992, "Reconstruction from Recollection and the Refutation of Idealism: A Kantian Theme in the Aufbau", Synthese, vol. 93, pp. 93-105.

Recibido el 11 de noviembre de 2008; aceptado el 11 de agosto de 2009. 